\title{
An assessment of growth hormone provocation tests
}

\author{
P C Hindmarsh, P G F Swift
}

There are a number of tests available for assessment of anterior pituitary hormone function. Recent concern has centred on the use of provocative tests for growth hormone release and in particular the glucagon and insulin induced hypoglycaemia or insulin tolerance test (ITT). These tests have been associated with cerebral oedema and death of at least two children and, as a result, questions have been raised as to the current management and investigation of children with potential abnormalities of growth hormone secretion. ${ }^{1}$

This review describes the background and theory behind the tests as well as an appraisal of some of the more common methods of assessing growth hormone release. It does not provide detailed information on the construction of 12 or 24 hour profiles of growth hormone secretion, as it is not felt that these are applicable to routine clinical investigation.

Central to the use of any test are its performance characteristics in terms of efficiency, sensitivity, and specificity. Attention must also be paid to the method used to measure growth hormone in the serum. This review attempts to assess the importance of these issues.

\section{Background}

A comprehensive assessment of anterior pituitary function requires the proper use and interpretation of well described combined function tests. ${ }^{2}$ Classical endocrine function tests consist of the administration of a stimulus known to produce a quantifiable response of the hormone thought to be deficient. For assessment of growth hormone and adrenocorticotrophic hormone (ACTH) insulin induced hypoglycaemia (ITT) has been for many years the 'gold standard'. The advantage of using the ITT is that a clearly described standardised stimulus is applied, a quantifiable hypoglycaemia stimulus is produced, and the endocrine responses have been defined in adults at least. As an assessment of the cortisol response to a stressful stimulus it is the only test that has been validated (against surgical stress). ${ }^{3}$ As with other provocation tests, the function of the gonadotroph and thyrotroph cells can be tested concurrently by the administration of their respective releasing hormones.

When should combined anterior pituitary function tests be performed and when only a growth hormone provocation test?
Epidemiological studies would suggest that idiopathic isolated growth hormone deficiency occurs more frequently than multiple pituitary hormone deficiency. It is not always possible, however, for the clinician to be sure that there is no other associated anterior pituitary hormone deficiency in addition to growth hormone insufficiency. Further, the loss of anterior pituitary hormones in multiple pituitary hormone deficiency most often starts with growth hormone. This pattern of loss may be of relevance in ascertaining the correct underlying diagnosis and whether other investigations such as a computed tomography or magnetic resonance imaging are required. Thyroxine and cortisol are important in the regulation of the growth hormone gene so that knowledge of thyroxine and cortisol status is essential before embarking on growth hormone testing. Thyroxine can be easily measured on a single blood specimen but the integrity of the pituitary-adrenal axis is more difficult to assess with a single random morning blood sample because of the pulsatility of cortisol. ${ }^{4}$ In practice most clinicians before embarking on growth hormone assessment in a slowly growing child would attempt to confirm adequate thyroid and cortisol concentrations as well as measuring a full blood count and assessing renal function. Antigliadin antibodies might be measured and a karyotype examined particularly in girls. Any doubt about the cortisol status makes a more comprehensive assessment in the specialised endocrine centre absolutely mandatory.

Growth hormone release is under the control of the two hypothalamic peptides, growth hormone releasing hormone (GHRH) and somatostatin. Recent work has suggested that endogenous GHRH is extremely important in determining the magnitude of the growth hormone secretory burst, whereas somatostatin is important in determining the timing of that event. ${ }^{5}$ The stimuli used to provoke growth hormone secretion probably act through either or both of these peptides. In man there is no clear evidence to determine upon which of the pathways the various stimuli act. Therefore, it is difficult to compare the results of individual tests, although it is generally accepted that a cut off growth hormone peak response of $7 \cdot 5-10 \mu \mathrm{g} / 1(15-20 \mathrm{mU} / \mathrm{l})$ is a reasonable value for determining whether an individual is growth hormone sufficient or insufficient. 
Table 1 Effect of applying different peak serum growth hormone values in response to ITT for diagnosis of growth hormone insufficiency using height velocity as standard

\begin{tabular}{llll}
\hline $\begin{array}{l}\text { Growth hormone } \\
\text { concentration } \\
(m U /)^{\star}\end{array}$ & $\begin{array}{l}\text { Efficiency } \\
(\%)\end{array}$ & $\begin{array}{l}\text { Sensitivity } \\
(\%)\end{array}$ & $\begin{array}{l}\text { Specificity } \\
(\%)\end{array}$ \\
\hline 10 & 63 & 51 & 79 \\
13.5 & 66 & 64 & 70 \\
15 & 67 & 69 & 63 \\
20 & 68 & 82 & 49 \\
\hline
\end{tabular}

^By immunoradiometric assay ${ }^{10} ; 1 \mu \mathrm{g} / \mathrm{l}=2.6 \mathrm{mU} / 1$.

Interpretation of growth hormone tests

The aim of any diagnostic test is to complement the clinical history and examination to the point where false positive and false negative diagnoses are reduced to a minimum. The sensitivity and specificity of the test in question give guidelines to the clinician as to how likely a correct answer confirming or refuting the diagnosis is likely to be. Sensitivity and specificity values of over $80 \%$ are generally accepted as indicating an acceptable test performance. Defining the gold standard is often difficult ${ }^{67}$ and the accepted cut off point for growth hormone concentrations remains debatable particularly when it is remembered that normal children may have very low peak values. ${ }^{8}$

If it is accepted that growth rate is the most important index against which growth hormone measurements should be compared, then the test parameters could be defined as follows: the efficiency of the test would be the percentage of all results which were true positive or negative - a negative result being when an arbitrary (but agreed) concentration is exceeded and a child is shown to have a normal growth velocity. $A$ positive result would be when the cut off value is not exceeded in a child with a subnormal height velocity. With these definitions, the sensitivity of the test would be calculated as the number of patients growing poorly with a positive test divided by the total number of children growing poorly. Specificity would be the number of children growing normally with a negative test, divided by the total number of children growing normally. Both values are expressed as percentages.

Few comparative studies have used growth rate as part of the definition for interpreting results. What has usually happened is that the ITT and other tests have been compared. Not surprisingly the ITT has scored extremely well because, being the standard, it is allocated a value of $100 \% !^{9}$ Table 1 provides an analysis of ITT performance against growth rate ${ }^{10}$ and

Table 2 Performance characteristics of tests of growth hormone secretory status compared with the ITT using a cut off value of $7.5 \mu \mathrm{g} /(15 \mathrm{mU} /)$

\begin{tabular}{lllll}
\hline & $\begin{array}{l}\text { Efficiency } \\
(\%)\end{array}$ & $\begin{array}{l}\text { Sensitivity } \\
(\%)\end{array}$ & $\begin{array}{l}\text { Specificity } \\
(\%)\end{array}$ & $\begin{array}{l}\text { Reference } \\
\text { No }\end{array}$ \\
\hline Sleep & & & & \\
$\quad$ Monitored by & & & & \\
$\quad$ EEG & 88 & 86 & 95 & 11 \\
$\quad$ Not monitored & 79 & 67 & 82 & 12 \\
Arginine & 72 & 73 & 85 & 13 \\
Clonidine & 70 & 70 & 85 & 14 \\
IGF-1 & 75 & 95 & 60 & 15 \\
IGFBP-3 & 96 & 97 & 95 & 15 \\
\hline
\end{tabular}

$\mathrm{EEG}=$ electroencephalography. table 2 shows the efficiency, sensitivity, and false negative rate of a number of tests published in the literature compared with the ITT. There is little to choose between any of the tests.

A number of reasons have been proposed to explain the discordance between the results obtained from growth hormone testing compared with prolonged observation of growth velocity in children. First, a raised growth hormone concentration at time 0 during the test leads to difficulty in interpretation. Tests are designed to evaluate the secretory pathway, particularly the readily releasable pool of growth hormone. If there has recently been a pulse of growth hormone and the pool is low, the subsequent response will be attenuated. ${ }^{13}$ Ideally, before any test, there should be a run in period where samples are obtained for at least 30 and preferably 60 minutes before the study in order that the response can be interpreted in the light of possible previous secretory episodes.

Second, children's growth varies with the seasons and there is evidence to suggest cycles of periodicity greater than one year. ${ }^{16}$ Growth hormone secretion is related to growth rate $e^{17-19}$ and it is possible that some of the discordance could be explained by the performance of the test during a period of relatively faster growth when growth hormone secretion was greater whereas annualised growth rate might indicate a different picture of overall growth failure. This highlights the difficulty of comparing one year of growth data with a one day stimulation test. Further, the relation between growth rate and growth hormone secretion is a continuum so any cut off value chosen to separate normality from abnormality is a compromise balancing sensitivity and specificity.

A third source of discordance is that a particular stimulus might release pituitary hormones when the problem is in the hypothalamus. It is important therefore to use stimuli which seem to act predominantly on the hypothalamus (for example, ITT, clonidine) rather than the pituitary gland (for example, GHRH).

Finally, the reproducibility of any test needs to be considered. A number of studies have demonstrated that the reproducibility of the physiological and pharmacological methods of assessing growth hormone secretion in an individual is poor. ${ }^{20-22}$ Interindividual and intraindividual variation is considerable and makes interpretation difficult (table 3).

Table 3 Within individual coefficients of variation for growth hormone tests (derived from Saini et $\mathrm{al}^{22}$ )

\begin{tabular}{ll}
\hline & $\begin{array}{l}\text { Coefficient of } \\
\text { variation }(\%)\end{array}$ \\
\hline Mean 24 hour growth hormone & \\
concentration & $35 \cdot 0$ \\
Urinary growth hormone & $30 \cdot 1$ (prepubertal) \\
Clonidine & $43 \cdot 9$ (pubertal) \\
Arginine & $58 \cdot 0$ \\
Levodopa & $77 \cdot 5$ \\
GHRH & $89 \cdot 2$ \\
Thyroxine & $60 \cdot 0$ \\
& $13 \cdot 0$ \\
\hline
\end{tabular}


Growth hormone tests

These are classically divided into physiological and pharmacological tests. Practical details of endocrine investigations are given by $\mathrm{Hughes}^{2}$ and what follows is a commentary on the indications for and recent ideas on testing for growth hormone insufficiency. As a general principle these investigations should be performed only in centres with personnel experienced in and dedicated to the performance of such investigations and in laboratories regularly performing internal and external quality assessment of assay methodology.

\section{(A) INDICATIONS FOR GROWTH HORMONE TESTING \\ 1. Inadequate growth velocity}

This is the prime indication for investigation. Short stature in itself is not an indication for testing and it is, therefore, important not to use growth hormone measurements or provocation tests as a screen for all individuals with short stature. The decision to investigate the growth hormone axis should be based on the knowledge that growth velocity is inadequate, that other explanations for growth failure have been excluded as far as possible, and that there is a real possibility of hypothalamic or pituitary pathology.

In practice, this means careful observation of growth and calculation of growth rate at an interval not less than six months and preferably 12 months. Growth rates vary from year to year ${ }^{16}$ but the following rules can be applied. If height velocity, measured over one year, is less than the third height velocity centile, investigation is warranted because the chances of the next year's growth rate being normal is only $3 \%$. For children whose growth rates are between the third and 50th centiles observation for a second year is advisable. The chance of a normal child growing for two successive years with a height velocity less than the 25 th centile is $0.25 \times 0.25=0.0625$. In other words, only $6 \cdot 25 \%$ of the children would be expected to yield normal results on investigation. The longer a velocity remains below the 50 th centile, the more likely it is that investigation will reveal an abnormality. ${ }^{23}$ The new national growth charts encompass this concept so that any child who crosses from one of the new centile divisions to another during the course of one year requires evaluation. ${ }^{24}$

\section{Neonatal problems}

Neonates or infants with prolonged jaundice and hypoglycaemia that might be associated with multiple pituitary hormone deficiency are candidates for investigation of growth hormone secretory status. This must be done only when thyroxine and cortisol deficiencies have been corrected. Extreme care needs to be taken in the performance of the growth hormone provocation tests and, if hypoglycaemia is present on the time 0 minute sample, no further stimulus is required. Hypoglycaemia should be an adequate stimulus and all that should be done is the collection of two samples separated in time by five to 10 minutes for assay of growth hormone.

\section{Growth failure following cranial irradiation for} brain tumours or leukaemia

Cranial irradiation for brain tumours is associated with growth failure, growth hormone insufficiency (in approximately $90 \%$ of children) and other anterior pituitary hormone deficiencies may also be present. The prevalence of growth hormone insufficiency after cranial irradiation for leukaemia is less clear. Investigation should be based on clinical observation of growth after radiotherapy with or without chemotherapy.

\section{Pubertal delay}

In certain situations, it may be difficult to ascertain whether a child who is growing slowly with constitutional delay of growth and puberty is going to have an adequate pubertal growth spurt. In such a situation, it may be valuable to perform a growth hormone provocation test following 'priming' with exogenous sex steroid before the start of the investigation. The aim of this investigation is to ascertain whether there is going to be sufficient growth hormone available during the puberty growth spurt. Priming has usually been performed in males by giving a single intramuscular injection of testosterone $(100 \mathrm{mg})$ three days before the investigation or in females by giving ethinyloestradiol or stilboestrol (1 $\mathrm{mg}$ twice a day for two days). The latter can actually be used quite satisfactorily in both sexes. ${ }^{825}$ Using high doses of ethinyloestradiol is unnecessary and causes nausea and headache.

\section{(B) PHYSIOLOGICAL TESTS OF GROWTH} HORMONE SECRETION

Serum growth hormone concentrations during the daytime are low and because growth hormone secretion is pulsatile there is no place for random serum growth hormone measurements. As a general principle growth hormone responses are blunted in all tests in the presence of hypothyroidism, cortisol excess, and obesity. Four types of assessment of physiological secretion can be performed.

\section{Exercise test}

The exercise test should be performed with a bicycle ergometer and relies on the generation of a standard amount of work. At best the test gives $20 \%$ false positive results ${ }^{2}$ but when poorly performed the diagnostic yield is very low with at least $20-30 \%$ falsely low growth hormone concentrations 25 minutes after completing the exercise.

\section{Sleep studies}

Sleep studies have been used to investigate physiological growth hormone secretion. These tests can be performed with or without 
electroencephalographic monitoring, the aim is to obtain blood samples for growth hormone, at 15-20 minute intervals during stage 3 and 4 sleep. Performance characteristics are shown in table 2. This type of study when performed regularly in specialised units is well accepted by children but places heavy demands on manpower. It should be stressed that the relationship between sleep and maximum growth hormone release is one of association rather than causality which may be affected in a number of disease situations, for example deprivation syndromes. ${ }^{2627}$

\section{Urinary growth hormone measurement}

Urinary growth hormone has been proposed as a non-invasive method of assessing growth hormone secretory status. ${ }^{28}$ With improved radioimmunoassay and enzyme linked immunosorbent assay (ELISA) techniques, increased assay sensitivity allows measurements in small volumes of urine. Variability is high and two or three overnight collections are advised to overcome this problem. Timed overnight samples are recommended but there appears to be little to be gained by expressing values in terms of creatinine excretion, which is also highly variable (coefficient of variation $27 \%$ ). ${ }^{29}$ Renal function needs to be assessed before the study is conducted. Normal ranges have been described, but there is considerable overlap between groups of children with different abnormalities of growth hormone secretion. ${ }^{29-32}$ This reduces the specificity and sensitivity of the test to some extent but urinary growth hormone measurement in some circumstances does perform well against conventional tests with efficiency of $90 \%$, sensitivity of $80 \%$, and specificity of $98 \% .^{29}$

4. Insulin-like growth factor-1 and binding proteins With the increasing availability of specific radioimmunoassays for insulin-like growth factor-1 (IGF-1), the possibility of using a single blood test to aid in the diagnosis of growth hormone insufficiency has been promulgated. Serum IGF-1 values are low in growth hormone insufficiency. However, normal IGF-1 values are low in early childhood resulting in poor discrimination from subnormal values in that age group. There is also considerable overlap of IGF-1 in patients with growth hormone sufficiency and insufficiency resulting in poor performance of the test (table 2).

The recent demonstration of IGF binding proteins and their dependence on a number of hormonal regulators has suggested a role for these proteins in the assessment of growth hormone secretory status. IGF binding protein-3 (IGFBP-3) is largely regulated by the circulating concentrations of growth hormone and might be used as an indicator of growth hormone secretory status. Blum et al have suggested that there is excellent specificity and sensitivity when IGFBP-3 measurements are used in a group of children with short stature. ${ }^{15}$ This measure deserves further exploration as it might reduce the investigation of growth hormone insufficiency to a single blood test and allow sequential measures to be made over the course of a year to compare with growth data. Falsely low values might be expected in states of growth hormone resistance and in liver disease.

\section{(C) PHARMACOLOGICAL TESTS OF GROWTH} HORMONE SECRETION

As a first principle all these tests require good venous access. A nurse and a doctor should be present throughout the procedure and facilities for resuscitation need to be available. All of the tests were standardised in adults after an overnight fast. Fasting from midnight is the ideal but young children should be encouraged to have a late night snack and infants should not starve for more than six hours. Drinks of water are allowed.

After insertion of an intravenous cannula a period of acclimatisation should be allowed. Whenever possible, the test should be started by drawing a sample of blood 15-30 minutes before the application of the stimulus. This sample together with the 0 minute sample gives an idea of the growth hormone secretory status after the cannula is inserted before the time that the study is carried out. High growth hormone values which are declining during this time period are often associated with a poor response to the stimulus.

\section{ITT (often combined with thyrotrophin releasing hormone and luteinising hormone releasing factor tests)}

The ITT $(0 \cdot 15 \mathrm{U} / \mathrm{kg}$ soluble insulin) should only be used in specialist units when there is a need to test the hypothalamo-pituitary-adrenal axis as well as the growth hormone axis. ${ }^{33} \mathrm{It}$ should only be performed under strict conditions of surveillance utilising safety precautions detailed previously. ${ }^{1}$ The ITT is contraindicated in children with a recent history of epilepsy or hypoglycaemic convulsions and special precautions must be taken in children suspected of having hypopituitarism.

The growth hormone response may be impaired if hypoglycaemia is inadequate. An adequate hypoglycaemic stimulus is when the blood glucose falls to $2 \cdot 2 \mathrm{mmol} / 1$ or less, if the child is symptomatic, or if there is a $50 \%$ reduction in the blood glucose concentration. ${ }^{34}$ Blood samples need to be drawn at 30 minute intervals for two hours.

Lunch should be provided for the child at the end of the test and the child should not be sent home until an adequate meal has been taken and observed for at least 90 minutes after the meal to make certain that there is no vomiting. The intravenous cannula should be left in situ until lunch has been assimilated. If there is any doubt about the child's wellbeing overnight observation is recommended.

\section{Glucagon stimulation test}

This test is often used in younger children. Although the usual recommended dose of 
glucagon is $0.5 \mathrm{mg}$ problems can be encountered. Rebound hypoglycaemia may result because glucagon is a potent insulin secretagogue. The timing of this is variable and if delayed beyond the end of the test at 180 minutes it is particularly dangerous. This test should not be considered as any easier to perform than the ITT and careful monitoring of blood glucose concentrations is essential. Current work suggests that a glucagon dose of $15 \mu \mathrm{g} / \mathrm{kg}$ is adequate to stimulate growth hormone and cortisol ( $M$ O Savage, personal communication).

Care needs to be exercised in interpreting the cortisol response to the glucagon test. Peak ACTH concentrations are significantly less during glucagon than during the ITT and are also delayed. ${ }^{35}$ Higher and more consistent cortisol levels are obtained after intramuscular administration than after the subcutaneous route. ${ }^{36} 37$

Problems with hypoglycaemia may arise particularly in young children if glycogen stores are depleted by an overnight fast before the administration of glucagon and as recommended above for the ITT, lunch must be seen to be absorbed after the test before the intravenous cannula is removed. The test is contraindicated when cortisol deficiency is suspected as glycogen stores are likely to be low.

\section{Clonidine stimulation}

This is a commonly used stimulus in a dose of $0.15 \mathrm{mg} / \mathrm{m}^{2}$ orally. The Health Service Human Growth Hormone Committee compared the clonidine test with the ITT. ${ }^{14}$ Oral administration of clonidine is associated with a reproducible peak serum clonidine concentration about 60 minutes after administration. Side effects include drowsiness, pallor, and sometimes a marked fall in blood pressure. The effects seem to be related to the age of the child and are particularly marked in adults. Therefore, care needs to be taken with this test and hypotension with the prolonged drowsiness may necessitate the child being observed for the remainder of the day. Despite these reservations this test is commonly used in paediatric practice. Performance characteristics are shown in table 2.

\section{Arginine stimulation}

Arginine is a recognised stimulus of growth hormone release. Arginine (arginine monohydrochloride) provokes release of growth hormone when infused intravenously. Given over a 30 minute period $(0.5 \mathrm{~g} / \mathrm{kg})$ the side effects of nausea and irritation at the infusion site are minimised. Performance characteristics of this test are shown in table 2 and care is also required as amino acids such as arginine, while having a direct action on the hypothalamus, are also insulin secretagogues.

\section{Levodopa}

Levodopa may be administered orally to stimulate growth hormone release. The side effect of nausea limits its use, although concomitant administration of propranolol minimises this. A peak response is not expected until 90 minutes after administration so that a long sampling period of $150-180$ minutes is required for full assessment.

\section{GHRH stimulation test}

This tests the readily releasable pool of growth hormone contained within the somatotroph. There is, however, some uncertainty regarding the amount of growth hormone released for a particular GHRH given dose. Supramaximal doses of GHRH $(1-2 \mu \mathrm{g} / \mathrm{kg})$ have been given as an intravenous bolus, probably in excess of the requirement for maximal release. This test is not recommended for routine clinical practice. It does not test the hypothalamo-pituitary axis and it is not, therefore, a useful 'screening' test.

\section{Growth hormone assays}

There has been a recent increase in the number of growth hormone assays available for the measurement of serum growth hormone concentration. These methods range from the traditional polyclonal methodology using radioimmunoassay to sophisticated monoclonal radioimmunometric assays. Sensitivity has been greatly improved by the use of enzyme linked or chemiluminescent systems. The variety of assays introduces further problems in the interpretation of growth hormone responses.

Assays employ different reaction matrices and are almost certain to use antisera with differing epitope specificity so that universal cut off values, for example $7.5 \mu \mathrm{g} / 1$ ( $15 \mathrm{mU} / \mathrm{l})$, may not be appropriate using different assay systems. Care must be exercised in interpreting results from different laboratories. The clinician needs to be aware of the method in current use, the precision profile of that assay, and how the laboratory performs within the UK external quality assurance scheme. Table 1 shows the results of using the Hybritech system for measuring growth hormone.

\section{Conclusions}

Growth hormone provocation tests should be used only after careful clinical assessment has confirmed inadequate growth velocity, other potential causes of growth failure have been excluded, and that there is a real possibility of hypothalamic or pituitary hormone deficiency. These tests should be performed only in centres where there is experience in performing and interpreting them. The clonidine test is the most useful for paediatric practice. ITT and glucagon tests should only be used in specialist centres.

There remains the question of whether any growth hormone test should be performed in the assessment of the slowly growing child. Although idiopathic isolated growth hormone insufficiency is one of the commonest endocrine causes of poor growth the clinical 
features are poor indicators of the diagnosis or whether there is an isolated or multiple pituitary deficiency. In the neonatal period and after cranial irradiation there are clearer indications for testing.

The authors consider that growth hormone measurements remain a valid investigation in order to provide biochemical support for subsequent growth hormone treatment. Despite the statistical constraints of the tests the likelihood that a slowly growing child is growth hormone insufficient is increased twofold in the presence of a positive test but only decreased by half in the presence of a negative one. Improving the probability of the presence of a growth hormone secretory disorder is particularly important if general practitioners are to be asked to prescribe and, therefore, to take some shared responsibility for the prescription. Also, detailed evaluation would seem to be a prerequisite for outcome audit and research purposes. Advances in cellular and molecular biology will gradually clarify the diagnosis of idiopathic isolated growth hormone insufficiency but these techniques will only be as good as the defined phenotype.

We thank the following paediatric endocrinologists for their help and advice in the preparation of this manuscript: C G D Brook, M D C Donaldson, CJ Kelnar, M O Savage, S Shalet, and $R$ Stanhope.

1 Shah A, Stanhope R, Matthew D. Hazards of pharmacological tests of growth hormone secretion in childhood. $B M F$ 1992; 304: 173-4.

2 Hughes IA. Handbook of endocrine investigations in children. London: Wright, 1986.

3 Plumpton FS, Besser GM. The adrenocortical response to surgery and insulin induced hypoglycaemia in cortisteroid treated and normal subjects. Br f Surg 1969; 56: 216-9.

4 Crowley S, Hindmarsh PC, Honour JW, Brook CGD. Assessment of cortisol secretion in children with asthma Assessment of cortisol secretion in children with asthma
treated with inhaled steroids. Pediatr Res 1993; 33 (suppl): treated with

5 Hindmarsh PC, Brain CE, Robinson ICAF, Matthews DR, Brook CGD. The interaction of growth hormone releasing hormone and somatostatin in the generation of a GH pulse. Clin Endocrinol (Oxf) 1991; 35: 353-60.

6 Sox HC Jnr. Probability theory in the use of diagnostic tests. Ann Intern Med 1986; 104: 60-6.

7 Pauker SG, Kopelman RI. Interpreting hoofbeats: can Bayes help clear the haze. $N$ Engl $\mathcal{f}$ Med 1992; 327: 1009-13.

8 Marin G, Domene HM, Barnes KM, et al. The effects of estrogen priming and puberty on the growth hormone response to standardized treadmill exercise and arginineinsulin in normal girls and boys. 7 Clin Endocrinol Metab 1994; 79: 537-41.

9 Rose SR, Ross JL, Uriarte M, Barnes KM, Cassorla FG, Cutler GB Jnr. The advantage of measuring stimulated as compared with spontaneous growth hormone levels in the diagnosis of growth hormone deficiency. $N \mathrm{Engl} \mathrm{f} \mathrm{Med}$ 1988; 319: 201-7.

10 Dattani MT, Pringle PJ, Hindmarsh PC, Brook CGD. What is normal stimulated growth hormone concentration? f Endocrinol 1992; 133: 447-50.

11 Hindmarsh PC, Smith PJ, Taylor BJ, Pringle PJ, Brook CGD. Comparison between a physiological and pharmacological stimulus of growth hormone secretion: response to stage IV sleep and insulin-induced hypoglycaemia. Lancet 1985; ii: 1033-5.

12 King JM, Price DA. Sleep-induced growth hormone release - evaluation of a sim

13 Youlton R, Kaplan SL, Grumbach MM. Growth and growth hormone. IV. Limitations of the growth hormone response to insulin and arginine in the assessment of
growth hormone deficiency in children. Pediatrics 1969; 43: 989-1004.

14 Health Services Human Growth Hormone Committee. Comparison of the intravenous insulin and oral clonidine tolerance tests for growth hormone secretion. Arch Dis tolerance tests for growth
Child 1981; 56: $852-4$.

15 Blum WF, Ranke MB, Keitzmann K, Grauggel E, Zeisel H, Bierich JR. A specific radioimmunoassay for the growth hormone $(\mathrm{GH})$-dependent somatomedin-binding protein: its use for diagnosis of GH deficiency. $\mathcal{F}$ Clin Endocrinol Metab 1990; 70: 1292-8.

16 Butler GE, McKie M, Ratcliffe SG. The cyclical nature of prepubertal growth. Ann Hum Biol 1990; 17: 177-98.
17 Hindmarsh PC, Smith PJ, Brook CGD, Matthews DR. The relationship between growth velocity and growth hormone secretion in short prepubertal children. Clin Endocrinol (Oxf) 1987; 27: 581-91.

18 Albertsson-Wikland $K$, Rosberg S. Analyses of 24-hour growth hormone profiles in children: relation to growth. $\mathscr{f}$ Clin Endocrinol Metab 1988; 67: 493-500.

19 Spadoni GL, Cianfarani S, Bernardini S, et al. Twelve hour spontaneous nocturnal growth hormone secretion in growth retarded patients. Clin Pediatr (Phila) 1988; 27: growth

20 Donaldson DL, Hollowell JG, Pan F, Gifford RA, Moore WV. Growth hormone secretory profiles. Variation on WV. Growth hormone secretory profiles. Var

21 Albertsson-Wikland K, Rosberg S. Methods of evaluating spontaneous growth hormone secretion. In: Ranke MB spontaneous growth hormone secretion. In: Ranke MB, ed. Functional endocrinologic diagnostics in child

22 Saini S, Hindmarsh PC, Matthews DR, et al. Reproducibility of 24-hour serum growth hormone profiles in man. Clin Endocrinol (Oxf) 1991; 34: 455-62. 23 Brook CGD, Hindmarsh PC, Healy MJR. A better to detect growth failure. $B M \mathcal{F}$ 1986; 293: 1186.

24 Cole TJ. Do growth chart centiles need a face-lift? $B M \mathcal{F}$ 1994; 308: 641-2.

25 Lippe B, Wong SR, Kaplan SA. Simultaneous assessment of growth hormone and ACTH reserve in children pretreated with diethylstilbestrol. F Clin Endocrinol Metab 1971; 33: 455-62.

26 Taylor B, Brook CGD. Sleep EEG in growth disorders. Arch Dis Child 1986; 61: 754-60.

27 Stanhope R, Adlard P, Hamill G, Jones J, Skuse D, Preece MA. Physiological growth hormone (GH) secretion MA. Physiological growth hormone (GH) secretion during the recovery from psychosocial dwarfi
report. Clin Endocrinol (Oxf) 1988; 28: 335-9.

28 Sukegawa I, Hizuka N, Takano K, et al. Measurement of nocturnal urinary growth hormone values. Acta Endocrinol (Copenh) 1989; 121: 290-6.

29 Skinner AM, Clayton PE, Price DA, Addison GM, Mui CYW. Variability in the urinary excretion of growth hormone in children: a comparison with other urinary proteins. F Endocrinol 1993; 138: 337-43.

30 Albini CH, Quattrin T, Vandlen RL, MacGillivray MH. Quantitation of urinary growth hormone in children with normal and abnormal growth. Pediatr Res 1988; 23: 89-92.

31 Walker JM, Wood PJ, Williamson S, Betts PR, Evans AJ. Urinary growth hormone excretion as a screening test for human growth hormone deficiency. Arch Dis Child 1990; 65: 89-92.

32 Phillip M, Chalew SA, Sten MA, Kowarski AA. The value of urinary growth hormone determination for assessment of urinary growth hormone determination for assessment of growth hormone deficiency and compliance with growth

33 Department of Health. Anterior pituitary function tests in childhood. London: DoH, 1992.

34 Gale EAM, Bennet T, MacDonald IA, Holst JJ, Matthews JA. The physiological effects of insulin-induced hypoglycaemia in man: responses at differing levels of blood glucose. Clin Sci 1983; 65: 263-71.

35 Littley MD, Gibson S, White A, Shalet SM. Comparison of the ACTH and cortisol responses to provocative testing with glucagon and insulin hypoglycaemia in normal subjects. Clin Endocrinol (Oxf) 1989; 31: 527-33.

36 Spathis GS, Bloom SR, Jeffcoate WJ, et al. Subcutaneous glucagon as a test of the ability of the pituitary to secrete

37 Rao RH, Spathis GS. Intramuscular glucagon as a provocative stimulus for the assessment of pituitary function; tive stimulus for the assessment of pituitary function; 36: 658-63.

\section{Commentary}

It is now more than 30 years since the induction of hypoglycaemia was described as a way of manipulating growth hormone secretion. ${ }^{1}$ The ITT, which can also provide an assessment of ACTH reserve, has been an established method to measure growth hormone secretion primarily in short children and stress related cortisol secretion primarily in adults with pituitary disorders. The Health Services Human Growth Hormone Committee was a government quango which was remarkably effective in maintaining a satisfactory standard of investigation for short children who might benefit from growth hormone treatment. ${ }^{2}$ When the government in its wisdom disbanded the quango, an internationally recognised UK database of adequately investigated children receiving growth hormone treatment soon disappeared. To its credit, the Kabi Pharmacia 